\title{
FAF1 induces neuronal death through cell-to-cell transmission
}

Gyeongrin Park

Bok-Seok Kim

Eunhee Kim

\section{Video Byte}

Keywords: Cell Communication and Signaling, FAF1, secretion, exosome, vesicle-free form, cell-to-cell transmission, cell death, FAS-associated factor 1, Parkinson's disease, propidium iodide, SH-SY5Y cells, brefeldin A, BFA, apoptosis, necrosis, signal peptides, endoplasmic reticulum, Golgi apparatus, secretory pathway, extracellular vesicles

Posted Date: November 12th, 2020

DOI: https://doi.org/10.21203/rs.3.rs-106651/v1

License: (c) (i) This work is licensed under a Creative Commons Attribution 4.0 International License. Read Full License 


\section{Abstract}

FAF1 is a protein involved in various biochemical processes including cell death, inflammation, and cell proliferation and is implicated in certain diseases, including cancer and Parkinson's disease. To date, FAF1 has been assumed to be locked within the cytosol-with no secretion mechanism reported for the protein. Now, researchers have discovered two mechanisms by which FAF1 can be secreted and transmitted between cells. Experiments on human neuroblastoma cells showed that FAF1 was secreted as cargo within exosomes, as well as in a free, non-exosomal form. Experiments also showed that FAF1 promoted the formation of exosomes, suggesting a regulatory role for the protein in exosome biogenesis. Additionally, extracellular FAF1 was transmitted to neighboring neuronal cells via endocytosis, triggering cell death through apoptotic and necrotic pathways. As the first to reveal these FAF1 secretion pathways this study could lead to ways of interfering with cell death by inhibiting FAF1 secretion. 